\title{
Design of Synchronized Supply Chains: A Six Sigma Tolerancing Approach
}

\author{
Y. NARAHARI \\ Computer Science and Automation \\ Indian Institute of Science \\ Bangalore - 560 012, India
}

\author{
N. VISWANADHAM \\ Mechanical and Production Engg \\ National University of Singapore \\ Singapore - 119260
}

\author{
R. BHATTACHARYA \\ Computer Science and Automation \\ Indian Institute of Science \\ Bangalore - 560012 , India
}

\begin{abstract}
A supply chain network can be viewed as a network of facilities in which a customer order will flow through internal business processes such as procurement, production, and transportation, ultimately reaching the required products on time to customers. The delivery performance of such a network can be maximized by pushing the work through the system in a way that the finished products reach the customer in a customer specified delivery window, with a very high probability. This entails synchronization and hence strict control of variability of deliveries at all intermediate points, to ensure that the raw materials and semi-finished work arrive at work spots in a timely fashion. In this paper, we explore the use of the Motorola six sigma tolerancing methodology to achieve synchronization in supply chains. In particular, we use the six sigma approach to: (1) analyze a given supply chain process for six sigma delivery performance; and (2) design synchronized supply chains to guarantee six sigma delivery performance. We provide an example of a plastics industry supply chain, for which we report analysis and design experiments that demonstrate the use of the six sigma approach in designing synchronized supply chains with high levels of delivery performance.
\end{abstract}

\section{Introduction}

In the simplest sense, the supply chain represents a process of delivering value to customers by creating and delivering products. Supply chains span from raw materials to manufacturing, distribution, transportation, warehousing, and product sales. The end goal of supply chain coordination is synchronization, or each member acting in ways that are appropriately timed with the actions of other supply chain members.

In this paper, we are concerned with how synchronization among internal processes in a supply chain can be achieved and built into a supply chain network, leading to what we call a synchronized supply chain. Towards this end, we invoke ideas from statistical tolerancing in general and the Motorola six sigma approach in particular. We believe synchronization is the key to achieving outstanding delivery performance and customer service levels in a supply chain (that is, guaranteeing a high probability of delivery within the promised delivery window or what we may call customer tolerance interval). Synchronization in addition will lead to reduction of lead times and inventory levels, thus contributing to overall cost reduction.

\subsection{Review of Relevant Work}

Combating various sources of uncertainty in supply chains has been studied by a number of researchers. Some of the important references include $[1,2,3]$. The role of variability reduction as a means of lead time reduction is a popular topic in the area of manufacturing systems [4] and in the area of business processes such as new product design and development [5]. This paper is concerned with how variability reduction leads to better synchronization in a supply chain. Synchronization is also the key theme behind the Just-in-Time (JIT) philosophy in manufacturing.

We explore the use of mechanical design tolerancing approaches towards designing synchronized supply chains. A survey of tolerance analysis and synthesis approaches can be found in $[6,7,8]$. The Motorola six sigma approach is described in $[9,10]$.

\subsection{Outline}

In this paper, we explore the use of the Motorola six sigma approach to:

- analyze a given supply chain process for six sigma delivery performance; and 
- design synchronized supply chains with six sigma delivery performance.

By six sigma delivery performance, we mean that the probability of delivering products to customers within a customer specified delivery window is at least as high as 0.9999966 [10].

In Section 2, we provide an overview of the Motorola six sigma approach to tolerancing. We bring out the notion of process capabilities and the meaning of six sigma performance. Section 3 presents an example of an aggregate-level supply chain in a plastics industry and we discuss analysis of lead time performance of this supply chain using six sigma terminology. In Section 4, we report on two different types of design studies on this supply chain. These are related to determining a pool of values for nominals and finding a variance pool.

\section{Six Sigma Approach to Toler- ancing: An Overview}

Six sigma quality is the benchmark of excellence for product and process quality, popularized by Motorola $[9,10]$. It provides a quantitative, statistical notion of quality useful in understanding, measuring, and reducing variation. A product is said to be of six sigma quality if there are no more than 3.4 non-conformities per million opportunities $(3.4 \mathrm{ppm})$ at the part and process-step level, in the presence of typical sources of variation. The six sigma quality concept recognizes that variations are inevitable due to insufficient design margin, inadequate process control, imperfect parts, imperfect materials, fluctuations in environmental conditions, operator variations, etc.

Tolerance analysis and synthesis in the six sigma program are based on six sigma characterization of products and processes. The process capability indices $C_{p}$ and $C_{p k}$ are used as the vehicles to characterize the product-process quality.

\section{Process Capability Indices}

Let $U$ and $L$ be the upper and lower specification limits, respectively, of a part or subassembly dimension in the case of mechanical assemblies. In the case of a supply chain process, they represent the maximum and minimum lead times tolerated for an individual business process or the overall supply chain process. In the sequel of this section, we will explain the concepts with relevance to mechanical assembly design.
Interpretation to the supply chain context is straightforward. Assume that $\sigma$ is the standard deviation of the process that produces the dimension. Then, the index $C_{p}$ is defined as:

$$
C_{p}=\frac{U-L}{6 \sigma}
$$

The numerator above represents the specification width whereas the denominator captures the total width of the $3 \sigma$ limits of the process distribution. For the rest of the discussion, assume that the process is normally distributed. The denominator then represents $99.73 \%$ limits for the process distribution. If $C_{p}=1$, the implication is that the specification width is the same as the distribution width and when the process mean is centered at $\left(\frac{U+L}{2}\right)$ without any shift, the probability that the actual dimension is within the specification limits is $0.9973(2700 \mathrm{ppm}$ defect rate). Similarly, if $C_{p}=2$, we have that the specification width is twice that of the distribution. In this case, when the process mean is centered at $\left(\frac{U+L}{2}\right)$ without any shift, the probability of conformance is 0.999999998 (.002 ppm defect rate). Since $\left(\frac{U-L}{2}\right)$ is the tolerance $T$ of the part dimension (or in general of any attribute of a product), we have that:

$$
\sigma=\frac{U-L}{6 C_{p}}=\frac{T}{3 C_{p}}
$$

The index $C_{p}$ does not capture how far away the process mean $\mu$ is from the ideal value $\tau$ (target value). The Motorola six sigma program assumes that the ideal value of the process mean is the midpoint of the specification interval, i.e. $\left(\frac{U+L}{2}\right)$. The index $C_{p k}$ captures the effect of the shift in the process mean in the following way:

$$
C_{p k}=C_{p}(1-k) \quad \text { where } k=\frac{|\tau-\mu|}{\left(\frac{U-L}{2}\right)}
$$

The factor $k$ above can be interpreted as the fraction of tolerance consumed by the mean shift. The above definition of $C_{p k}$ assumes that $\tau=\frac{U+L}{2}$ and for a general definition, refer [9].

The Motorola convention is to use a one sided mean shift of $1.5 \sigma$. The one sided mean shift is perhaps motivated by common physical phenomena such as tool wear. If $C_{p}=2$ and $C_{p k}=1.5$ (mean shift consumes 25 percent of the tolerance range), the probability of conformance can be shown to be 0.9999966 , which is equivalent to $3.4 \mathrm{ppm}$. Thus $C_{p} \geq 2$ and $C_{p k} \geq 1.5$ imply six sigma quality, assuming a $1.5 \sigma$ one sided mean shift. $C_{p} \geq 1$ and $C_{p k} \geq 0.5$ refer to three sigma quality, assuming a $1.5 \sigma$ one sided mean shift. 
The Motorola program assumes a relationship of the form

$$
Y=a_{0}+a_{1} X_{1}+a_{2} X_{2}+\ldots+a_{n} X_{n}
$$

If there is no mean shift, then the following formulae (called the Root Mean Square or RSS formulae) are applicable.

$$
\begin{aligned}
& \mu_{Y}=a_{0}+a_{1} \mu_{1}+a_{2} \mu_{2}+\ldots+a_{n} \mu_{n} \\
& \sigma_{Y}^{2}=a_{1}^{2} \sigma_{1}^{2}+a_{2}^{2} \sigma_{2}^{2}+\ldots+a_{n}^{2} \sigma_{n}^{2}
\end{aligned}
$$

Recall that $\sigma_{i}$, for $i=1, \ldots, n$, can also be written as:

$$
\sigma_{i}=\frac{T_{i}}{3 C_{p i}}
$$

where $T_{i}$ is the tolerance range of the $i$ th part and $C_{p i}$ is the $C_{p}$ value for the $i$ th part $(i=1, \ldots, n)$. In the presence of a mean shift, the six sigma program suggests the use of the Dynamic RSS method, where the $C_{p k}$ values, $C_{p k 1}, \ldots, C_{p k n}$, of the individual processes corresponding to dimensions $X_{1}, \ldots, X_{n}$; and the tolerances, $T_{1}, \ldots, T_{n}$, of the individual parts, are used in the following way to compute the variance of $Y$ :

$$
\sigma_{Y}^{2}=a_{1}^{2}\left(\frac{T_{1}}{3 C_{p k 1}}\right)^{2}+\ldots+a_{n}^{2}\left(\frac{T_{n}}{3 C_{p k n}}\right)^{2}
$$

Note that the standard deviations $\sigma_{i}$ are inflated by an amount equal to $\frac{C_{p i}}{C_{p k i}}$, for $i=1, \ldots, n$. Thus the dynamic RSS method emulates random behavior in the process mean by inflating the process standard deviation.

Tolerance synthesis employs the approach of using tolerance analysis in an iterative way. Each iteration will evaluate the resulting probability of non-conformance and the $C_{p}$ and $C_{p k}$ values. The synthesis procedure seeks to obtain a probability of non-conformance of at most $3.4 \mathrm{ppm}$, which is guaranteed by $C_{p} \geq 2$ and $C_{p k} \geq 1.5$. The synthesis can assume several forms: finding optimal values for nominal dimensions; finding optimal values for tolerances; and establishing a variance pool that can be allocated to individual processes so as to obtain the desired assembly yield.

\section{An Example}

We now consider a supply chain for a plastics industry (a certain anonymous firm in the western state of Maharashtra, India) and provide the basis and notation for applying statistical tolerancing concepts. The supply chain process in question has six business processes:
1. Procurement: The chemicals and other raw materials that are used in the manufacturing of the plastic are procured in this stage. Typically, several suppliers are involved. At an aggregate level, we will assume one mega supplier. Let $X_{1}$ denote the procurement lead time.

2. Sheet Fabrication: Here, from the chemicals and other raw materials, thin sheets of plastic are fabricated at a fabrication plant. These sheets are then transported to another plant for manufacturing and assembly. Let $X_{2}$ be the sheet fabrication lead time.

3. Transportation: The thin plastic sheets fabricated in the earlier stage are transported in trucks to a manufacturing and assembly facility. Assume that the logistics lead time here is $X_{3}$.

4. Manufacturing: In the manufacturing and assembly plant, numerous types of components are manufactured from the plastic'sheets. This is a multistage process and the processing depends on component types. We will aggregate this business process into a single stage and call the manufacturing lead time as $X_{4}$.

5. Assembly: In the same manufacturing and assembly facility, the components produced are assembled into different types of customer-desired products. Customization also happens here. We assume the assembly lead time to be $X_{5}$.

6. Delivery: Warehouses are located in the manufacturing and assembly plant itself. The final products are stacked here and supplied directly to customers against their orders. Third party logistics providers are used here. Let $X_{6}$ be the delivery time to a certain pool of customers who are co-located.

For the above system, we postulate that JIT philosophy is used and there is negligible waiting between one business process and the next one. The supply chain process lead time, $Y$, can then be described as:

$$
Y=\sum_{i=1}^{6} X_{i}
$$

Note that $X_{i} \mathrm{~s}(i=1, \ldots, 6)$ are mutually independent continuous random variables and hence $Y$ is also a continuous random variable. Furthermore, if $X_{i} \mathrm{~S}$ are normally distributed, then $Y$ is also normally distributed. 
Table 1: Notation for different measures $(i=1, \ldots, 6)$

\begin{tabular}{ll}
\hline \hline & \\
$B P_{i}$ & Business process $i$ \\
$X_{i}$ & Lead time of $B P_{i}$ \\
$\tau_{i}$ & Target value or nominal of $X_{i}$ \\
$T_{i}$ & Tolerance of $X_{i}$ \\
$\mu_{i}$ & mean lead time produced by $B P_{i}$ \\
$\sigma_{i}$ & standard deviation of lead time $X_{i}$ \\
$U_{i}$ & Upper Specification limit of $X_{i}$ \\
$L_{i}$ & Lower Specification limit of $X_{i}$ \\
$\left(L_{i}, U_{i}\right)$ & Delivery window for $B P_{i}$ \\
$C_{p i}$ & $C_{p}$ index for the process $B P_{i}$ \\
$C_{p k i}$ & $C_{p k}$ index for the process $B P_{i}$ \\
$Y$ & Supply chain process lead time $=\sum_{i=1}^{i=6} X_{i}$ \\
$\tau_{Y}$ & Nominal or target value of $Y$ \\
$T_{Y}$ & Tolerance of $Y$ \\
$\mu_{Y}$ & mean of $Y$ \\
$\sigma_{Y}$ & standard deviation of $Y$ \\
$U_{Y}$ & Upper Specification limit of $Y$ \\
$L_{Y}$ & Lower Specification limit of $Y$ \\
$\left(L_{Y}, U_{Y}\right)$ & Delivery window for the supply chain process \\
$C_{p Y}$ & $C_{p}$ index for the supply chain process \\
$C_{p k Y}$ & $C_{p k}$ index for the supply chain process \\
\hline \hline
\end{tabular}

Table 1 shows the notation we will use in the rest of the paper. Table 2 shows the typical nominals, tolerances, and standard deviations for the $X_{i}$ s. The standard deviations have been computed from the tolerances by assuming $C_{p}=1$ (three sigma performance) for each individual process. The standard deviation values will be halved when we assume $C_{p}=2$. Using the RSS formula, the standard deviation $\sigma_{Y}$ of the supply chain process can be computed easily as the square root of the sum of squares. In this case, $\sigma_{Y}=2.135$ days. Table 3 shows the specification range, tolerance, and the tolerance interval for $Y$ for different specified values of $C_{p}$ and $C_{p k}$. They are computed using the following formulae:

$$
\begin{gathered}
U_{Y}-L_{Y}=6 \sigma_{Y} C_{p Y} \\
T_{Y}=\frac{U_{Y}-L_{Y}}{2} \\
\text { Tolerance Interval }=\left(\tau_{Y}-T_{Y}, \tau_{Y}+T_{Y}\right)
\end{gathered}
$$

We have chosen $\tau_{Y}=83$ days, which is simply the sum of the nominals of the cycle times of the six business processes.

From Table 3, it is clear that for the given supply chain process to be six sigma, the specification range should
Table 2: Nominals, tolerances, and standard deviations (all values in Days)

\begin{tabular}{lllll}
\hline \hline & Lead Time & $\tau_{i}$ & $T_{i}$ & $\sigma_{i}$ \\
& & & & \\
$X_{1}$ & Procurement & 7 & 1 & 0.334 \\
$X_{2}$ & Sheet fabrication & 30 & 3 & 1.0 \\
$X_{3}$ & Transportation & 3 & 1 & 0.334 \\
$X_{4}$ & Manufacturing & 30 & 5 & 1.667 \\
$X_{5}$ & Assembly & 10 & 2 & 0.667 \\
$X_{6}$ & Delivery & 3 & 1 & 0.334 \\
& & & & \\
\hline \hline
\end{tabular}

Table 3: Tolerances and specification ranges for $Y$ (all values in Days)

\begin{tabular}{llll}
\hline \hline & & & \\
$\left(C_{p Y}, C_{p k Y}\right)$ & $U_{Y}-L_{Y}$ & $T_{Y}$ & Window \\
& & & \\
$(0.5,0)$ & 6.405 & 3.2025 & $(79.8,86.2)$ \\
$(1.0,0.5)$ & 12.81 & 6.405 & $(76.6,89.4)$ \\
$(1.5, .09375)$ & 19.215 & 9.61 & $(73.4,92.6)$ \\
$(2.0,1.5)$ & 25.62 & 12.81 & $(70.2,95.8)$ \\
\hline
\end{tabular}

be very wide, namely $(70.2,95.8)$. That is if the customers are prepared to tolerate such a wide window for delivery, then the process becomes six sigma even though the individual processes have been assumed to be three sigma processes. If the customers' tolerance interval is $(76.6,89.4)$, then the supply chain process becomes a three sigma process. If for the same tolerance range, the supply chain process is to be six sigma, then it would imply that the standard deviations of individual processes should be cut to half their original values, which means the individual processes themselves should be six sigma.

\section{Design Optimization for Six Sigma Performance}

Here, we describe two different types of design experiments. In the first, we determine the range of nominal values for the lead times of designated internal business processes, so as to achieve six sigma delivery performance. In the second, we compute a variance pool for lead times of designated business processes 
(hence their process capabilities) to achieve six sigma delivery performance.

\subsection{Finding a Nominal Pool}

Table 4 shows the structure of this design problem. In this problem, the tolerance $T_{Y}$ of the supply chain process is given. The tolerances for the lead times of individual processes are given as also the nominals of lead times of some of the business processes (say, $\left.\tau_{2}, \tau_{4}, \tau_{5}\right)$. The problem is to compute a range of values for the other nominals (in this case, $\tau_{1}, \tau_{3}, \tau_{6}$ ), so as to achieve six sigma performance. As an example, let us say we know the nominals of the fabrication lead time, manufacturing lead time, and assembly lead time; and we are to obtain a range of values of nominals for the procurement lead time, transportation lead time, and the logistics lead time. The first of these has implications on choice of suppliers, while the second and third influence the choice of logistics providers and location of suppliers. We can solve the problem through the following algorithm:

1. Assume appropriate process capabilities for individual business processes. Since we know the tolerances $T_{i}$, we can compute the standard deviations $\sigma_{i}$ using the RSS method or the dynamic RSS method.

2. Compute $\sigma_{Y}$ as the root of the sum of squares of $\sigma_{i}$ 's.

3. Knowing $T_{Y}$ and $\sigma_{Y}$, compute the range of values of nominals in the set $A-B$ over which six sigma delivery performance is guaranteed.

4. The pool of nominal values obtained in the previous step can be distributed to individual business processes based on engineering judgment and any other available information.

For example, let $\tau_{Y}=83$ days; $T_{Y}=6$ days; $T_{1}=$ $1 ; \quad T_{2}=3 ; \quad T_{3}=1 ; \quad T_{4}=3 ; \quad T_{5}=2 ; \quad T_{6}=1$. Let the nominal value of fabrication lead time $\left(\tau_{2}\right)$ be known to be 30 days; that of manufacturing lead time $\left(\tau_{4}\right)$ to be 30 days; and that of assembly lead time $\left(\tau_{5}\right)$ to be 10 days. It is required to find a range of values for the pool of other nominals $\tau_{1}+\tau_{3}+\tau_{6}$ such that the probability of delivery is at least 0.9999966 within the delivery window. To solve this problem, we first assume that all the individual processes are six sigma and compute individual standard deviations. To be conservative and realistic, we assume shifts and drifts in the mean $\left(1.5 \sigma_{i}\right)$, thus we use $C_{p k i}=1.5$ to compute
Table 4: Finding a nominal pool

Given:
1. $\tau_{Y}$, the target value for $Y$
2. $T_{Y}$, the tolerance range for $Y$
3. $T_{i}(i=1, \ldots, 6)$
4. A subset $B$ of $A=\left\{\tau_{1}, \ldots, \tau_{6}\right\}$
To Compute:
Range of values for all nominals in the set $A-B$
over which the supply chain process exhibits six
sigma delivery performance

the standard deviations. Then we compute $\sigma_{Y}$. It is found that as long as $\tau_{1}+\tau_{3}+\tau_{6}$ is in the range $(12.5,13.5)$, we obtain six sigma delivery performance. That is, for this range of values, the probability of $Y$ to be in the range $(77,89)$ is at least .9999966 . The maximum probability is attained at $\mathbf{1 3 . 0}$ days. We can choose any value that is convenient in this range. For example, the value 13.5 days provides us the most flexibility. This can be allocated to $\tau_{1}, \tau_{3}$, and $\tau_{6}$ in any possible way, based on best engineering judgment and any other technical considerations.

\subsection{Finding a Variance Pool}

Table 5 shows an outline of this problem. In this problem, the nominal value of the overall process, $\tau_{Y}$, as also the nominal values for the individual business processes are given. The tolerance $T_{Y}$ of the overall process and also that of some individual business processes are given. The problem is to find a variance pool that can be distributed across the individual business processes. How we distribute the variance pool can depend on our knowledge of the processes and best engineering judgment. This problem has implications on choice of suppliers, logistics providers, machining equipment, etc. For example, let the target values be: $\tau_{Y}=83$ days; $\tau_{1}=7 ; \quad \tau_{2}=30 ; \quad \tau_{3}=$ $3 ; \quad \tau_{4}=30 ; \quad \tau_{5}=10 ; \quad \tau_{6}=3$. Also, assume that $T_{2}=3 ; T_{4}=3 ; T_{5}=2$. If it is known that the above three processes (fabrication, manufacturing, and assembly) are six sigma, then the standard deviations can be computed by using the value of $C_{p k}$ which in this case is 1.5. This will take into account shifts and drifts in the mean value of the processes (Dynamic 
Table 5: Finding a variance pool

Given:

1. $\tau_{Y}$, the nominal value of $Y$

2. $T_{Y}$, the tolerance of $Y$

3. A subset $B$ of $A=\left\{T_{1}, \ldots, T_{6}\right\}$

4. $\tau_{i}(i=1, \ldots, 6)$, the nominal values of $X_{i}$

To Compute:

A variance pool for standard deviations

of business processes in the set $A-B$.

RSS). This leads to:

$$
\sigma_{i}=\frac{T_{i}}{4.5} ; i=2,4,5
$$

Now assume $T_{Y}$ to be 6 days. If we require that the the delivery performance be six sigma, we need $C_{p Y}=2.0$ and $C_{p k Y}=1.5$. Using this, we can compute the standard deviation $\sigma_{Y}$ for the supply chain process lead time. We will then get the variance pool, $\sigma_{1}^{2}+{\sigma_{3}}^{2}+{\sigma_{6}}^{2}$, for the three processes, viz. procurement; transportation, and delivery as 0.32 . This variance pool can then be distributed across the individual processes in any way guided by engineering judgment. For different values of $T_{Y}$, we will get different variance pools. For example, if $T_{Y}=7$ days, then the variance pool is 1.01 ; if $T_{Y}=8$ days, then the variance pool is 2.11 ; and so on.

\section{Conclusions}

Synchronization among internal business processes of a supply chain is a key to achieving a high level of delivery performance in supply chains. In this paper we have shown how the Motorola six sigma approach can be used in designing synchronized supply chains. We believe the paper is an important contribution towards applying statistical tolerancing techniques and best practices to design supply chain networks with high levels of delivery performance. There is available a rich variety of statistical tolerancing methodologies and best practices [8] and a natural direction for further work is to look into the application of those in designing synchronized supply chains.

In this article, we have only described (in Section 4) two possible design experiments. There are a rich variety of such design experiments we can conduct using the six sigma approach and other statistical tolerancing approaches and best practices. Also, we have implicitly assumed all distributions to be normal. Further the case study has been addressed at a coarse level of detail. There is available a rich variety of more general statistical tolerancing methodologies and best practices [8] and a natural direction for further work is to look into the application of those in designing synchronized supply chains.

\section{References}

[1] N. Viswanadham. Analysis of Manufacturing Enterprises. Kluwer Academic Publishers (In Press), 1999.

[2] H.L. Lee, V. Padmanabhan, and S.J. Whang. Information distortion in a supply chain: The bullwhip effect. Management Science, 43(4):546-558, April 1997.

[3] L.M. Corbett. Delivery windows: A new view on improving manufacturing flexibility and on-time delivery performance. Production and Inventory Management, 33(3):74-79, 1992.

[4] W.J. Hopp, M.L. Spearman, and D.L. Woodruff. Practical strategies for lead time reduction. Manufacturing Review, 3(2):78-84, 1990.

[5] Y. Narahari, N. Viswanadham, and V. Kiran Kumar. Lead time modeling and acceleration of product design and development. IEEE Transactions on Robotics and Automation, To appear: 1999.

[6] D.H. Evans. Statistical tolerancing: The state of the art, Part II. Method for estimating moments. Journal of Quality Technology, 7:1-12, 1975.

[7] D.H. Evans. Statistical tolerancing: The state of the art, Part III. Shifts and drifts. Journal of Quality and Technology, 7(2):72-76, 1975.

[8] Y. Narahari, R. Sudarsan, K.W. Lyons, M.R. Duffey, and R.D. Sriram. Design for tolerance of electro-mechanical assemblies: An integrated approach. IEEE Transactions on Robotics and Automation, To appear: 2000.

[9] M.J. Harry. The nature of six sigma quality. Technical report, Government Electronics Group, Motorola Inc., Scottsdale, Arizona, 1987.

[10] M.J. Harry and R. Stewart. Six sigma mechanical design tolerancing. Technical report, Government Electronics Group, Motorola Inc., Scottsdale, Arizona, 1988. 